\title{
Subword Recognition in Historical Arabic Documents using C-GRUs
}

\author{
Hanadi Hassen ${ }^{1}$, Somaya Al-Madeed ${ }^{1}$, Ahmed Bouridane ${ }^{2}$ \\ ${ }^{1}$ Computer Science and Engineering department, College of Engineering, Qatar University, Doha, Qatar \\ ${ }^{2}$ Department of Computer and Information Sciences, Northumbria University, \\ Newcastle upon Tyne, NE1 8ST, United Kingdom
}

\begin{abstract}
The recent years have witnessed an increased tendency to digitize historical manuscripts that not only ensures the preservation of these collections but also allows researchers and end-users' direct access to these images. Recognition of Arabic handwriting is challenging due to the highly cursive nature of the script and other challenges associated with historical documents (degradation etc.). This paper presents an end-to-end system to recognize Arabic handwritten sub words in historical documents. More specifically, we introduce a hybrid CNN-GRU model where the shallow convolutional network learns robust feature representations while the GRU layers carry out the sequence modelling and generate the transcription of the text. The proposed system is evaluated on two different datasets, IBN SINA and VML-HD reporting recognition rates of $96.10 \%$ and $\mathbf{9 8 . 6 0 \%}$ respectively. A comparison with existing techniques evaluated on the same datasets validates the effectiveness of our proposed model in characterizing Arabic subwords.
\end{abstract}

Keywords - handwriting recognition, Arabic historical documents, CNNs, GRUs, classification.

DOI: 10.18421/TEM104-19

https://doi.org/10.18421/TEM104-19

Corresponding author: Hanadi Hassen, Computer Science and Engineering department, College of Engineering, Qatar University, Doha, Qatar.

Email: $\underline{\text { hm1409611@qu.edu.qa }}$

Received: 10 August 2021.

Revised: 27 September 2021.

Accepted: 06 October 2021.

Published: 26 November 2021.

(c) BY-NC-ND_C 2021 Hanadi Hassen, Somaya Al-Madeed \& Ahmed Bouridane; published by UIKTEN. This work is licensed under the Creative Commons AttributionNonCommercial-NoDerivs 4.0 License.

The article is published with Open Access at www.temjournal.com

\section{Introduction}

Historical Arabic manuscripts carry substantial cultural significance. Consequently, a number of efforts have been made to digitize such collections hence ensuring their digital preservation. Nevertheless, simple digitization of such manuscripts limits their usage for researchers and end users if such collections are not accompanied by search engines allowing searching within the digitized content. The writing style in these documents vary significantly as a function of the geographical and temporal factors. The geographical factors relate to the location where the manuscript was produced while the temporal factors refer to the time or the era in which the writing was produced. From the perspective of Arabic handwriting, there exist a number of calligraphic styles, Kufic being the oldest one.

Arabic is a highly cursive script where characters are joined to form subwords. With 28 different characters in the alphabet, shape of a character is a function of its position within the subword. Many characters share shape and are differentiated by the number and position of dots. Some of the Arabic manuscripts lack the dots because they were written before the period of adding dots and diacritical marks to the Arabic script. On the other hand, many manuscripts that were written after the introduction of dots lost these markings due to physical degradations, thus contributing to the difficulties in automatic word recognition in such documents. As a result of such problems, word recognition in historical documents is much more challenging as compared to recognition of words in modern documents which have well-positioned markings [1].

Handwriting recognition techniques are typically categorized into segmentation-free (holistic) and segmentation-based (analytical) approaches. Segmentation-based techniques rely on segmenting the words into primitive units (subwords or characters) for recognition (Figure 1-a) while segmentation-free techniques employ words as units of recognition (Figure 1-b). 
a)
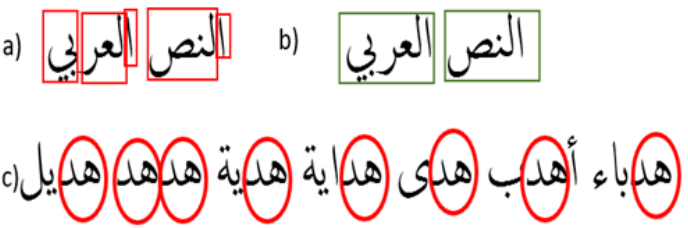

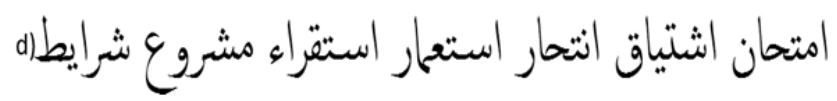

Figure 1. 1. a) Arabic subwords. b) Complete Arabic words c) Arabic words with some shared subwords

d) Few Arabic words that are used in other non-Arabic languages

Recognition of subwords has several advantages over holistic word recognition. Firstly, since subwords are disconnected parts of a word, it is easier to extract them as compared to complete words especially in case of cursive scripts like Arabic. Secondly, same subwords are shared across multiple words (Figure 1-c) and the total number of unique subwords is much less than the total number of unique words. In other words, the size of the dictionary of complete words is larger than that of the subwords, hence using subwords as units of recognition reduces the number of unique classes to be recognized. Another advantage of subword recognition is that it can be extended for handwriting recognition in other languages that share the same script as Arabic e.g., Urdu, Persian, etc. Few examples of the words shared between Arabic and Persian are shown in Figure 1-d. Finally, subword recognition is known to report higher recognition rates as opposed to word recognition [2].

This paper proposes a deep learning-based technique to recognize a substantial vocabulary of Arabic handwritten subwords extracted from historical documents. More specifically, we propose a hybrid CNN-GRU model that learns to discriminate between a large number of subword classes. The key highlights of this study are listed in the following.

An end-to-end system is proposed for recognition of challenging Arabic handwritten subwords extracted from historical manuscripts.

- A hybrid CNN-GRU architecture is introduced with shallow convolutional layers extracting robust features from subwords while the GRU layers learn to map the feature sequences to subword class labels.

- Experimental study is carried out on two benchmark datasets IBN SINA and VML-HD and recognition rates outperforming current state-of-the-art are reported.

- Unlike previous studies which consider only a subset of VML-HD dataset, the complete dataset has been employed for evaluation purposes in our experiments. To the best of our knowledge, no previous study has considered such diverse subword classes to evaluate the proposed models.
We organize this paper as follows. We first present an overview of notable studies to recognition of historical manuscripts in Section 2. Section 3 presents the details of the proposed technique while Section 4 introduces the experimental protocol, the reported results and the accompanying discussion. Finally, we conclude the paper with a summary of key findings in Section 5.

\section{Related Work}

The scientific literature on Arabic handwriting recognition is relatively limited especially in case of historical manuscripts [3]. Major proportion of the current state-of-the-art focuses on contemporary Arabic handwritten documents. Likewise, the literature is quite rich when it comes to analysis of historical manuscripts in the Roman script. We present, in this section, an overview of the research targeting Arabic historical manuscripts especially from the perspective of deep learning-based solutions. Furthermore, for completeness, Arabic handwriting recognition in contemporary documents is also discussed.

\subsection{Handwriting Recognition in Historical Arabic Documents}

A number of benchmark datasets have been made available to evaluate handwriting recognition (and other related tasks) on historical Arabic documents. Among these, HADARA [4], IBN-SINA [5], and VML-HD [6] have been commonly employed in a number of studies. Most of the work on HADARA dataset is on keyword spotting where the objective is to match a query word image in the collection of documents and retrieve all instances of the queried keyword. The IBN-SINA dataset is one of the most widely employed Arabic datasets and has been used for evaluation of word spotting as well as word recognition systems. VML-HD is a relatively recent dataset that contains a large vocabulary of subwords written in different writing styles.

Among notable studies on analysis of Arabic manuscripts, [7] propose a 5-layered convolutional neural network to recognize 68 classes of Arabic subwords extracted from historical collections. The network comprises 2 convolutional and 3 fully connected layers and reports a recognition rate of $81 \%$. The work was later extended in [8] to study the impact of synthesizing and augmenting data to recognize 39 subword classes from 10 pages of the VML-HD dataset. Through a comprehensive series of experiments, the authors concluded that data augmentation results in relatively better performance as compared to synthesizing data. Authors also concluded that 10 handwritten pages proved to be sufficient for training the model and recognizing the subwords. 
[9] proposed an efficient method to reduce the Arabic subword lexicon exploiting the topology and geometry of subwords. The skeleton of a subword image is extracted and is represented by a directed acyclic graph (DAG). The subword DAG is then encoded into a topological signature vector (TSV) which is a low dimensional feature vector. Given a query subword, the lexicon is reduced by computing the TSV distance between the query and the lexicon subwords and keeping a set of nearest subwords only. Subsequently, the reduced lexicon is fed to another nearest neighbour classifier. Experimental study of the system is carried out on the IBN-SINA database and recognition rates of $82.20 \%$ and $86.16 \%$ are reported with and without lexicon reduction respectively. Authors attributed the errors in case of lexicon reduction to the differences in writing styles of different writers.

In another study, the concept of topological signature vector (TSV) introduced in [1] was extended and improved to a weighted TSV (W-TSV) [10]. The introduction of weights proved effective as noise corresponds to smaller weights and thus it can be filtered out easily. The representation was also enriched by introducing three different DAG representations. These included the topological DAG (T-DAG) which preserves the information of the subword shape topology, the length DAG (L-DAG) which adds information about skeletal curve lengths by applying weights to the T-DAG and finally, the curvature DAG (C-DAG) which carries information about the curved structure of the subword. In a further extension of this work, the authors introduced the concept of Arabic word descriptors (AWD) [10] to create an index for a reference database of the subwords' shapes. The AWD is created from sorted and normalized structural descriptors (SD) of all the subwords using the bag-of-words (BOW) model, hence allowing an efficient shape matching. When a subword is provided as query, its reduced lexicon is acquired from the labels of the top ranked entries in the indexed database. The proposed technique improved the lexicon reduction accuracy on IBNSINA and IFN/ENIT [11].

Among other studies, skeleton-based features are employed for Arabic subword recognition with SVM classifier in [12] and an accuracy of $89.66 \%$ is reported in this study. Fouladi et al. [13] employ localized orientation histograms together with a contour alignment method to recognize subwords in a writer-dependent framework. Experiments on Arabic subwords in the IBN-SINA dataset as well as on a collection of Farsi words reported an accuracy of around $91 \%$.

A number of studies targeting IBN-SINA dataset are primarily concerned with dictionary reduction. The key idea is addressing the problem of high computational complexity due to very large lexicon. The results suggest that when performing lexicon reduction, a reduction of the dictionary would result in a reduced recognition accuracy. Hence, the tradeoff between high accuracy and reducing the lexicon size needs to be managed [10]. The performance charts for the IBN-SINA dataset are mostly dominated by conventional methods that employ handcrafted features with traditional classifiers such as the Nearest Neighbor. Feature learning and end-toend deep learning-based solutions have not been extensively used for recognition of Arabic historical documents.

\subsection{Handwriting Recognition in Modern Arabic Documents}

Recognition of Arabic handwritten words from contemporary documents has been thoroughly investigated in the literature. One of the earliest Arabic Handwriting Recognition (AHR) systems using deep learning was introduced in [14] where the well-known LeNet model was employed. The network consists of two stages, the first one recognizes characters while the second one recognizes the dots. In [15], the authors propose a LeNet-like architecture to extract features from Arabic words which are then fed as input to Hidden Markov Models for classification. Authors also compare the performance of machine-learned and hand-crafted features and the reported results suggest that the features extracted through $\mathrm{CNN}$ model outperform the hand-engineered features.

[16] proposed a multistage cascading method for recognizing handwritten Arabic text. Their approach begins by dividing the IFN/ENT database into partially interconnected clusters using the Hierarchical Agglomerative Clustering (HAC). The members of the cluster are then ranked by Pyramid Histogram of Oriented Gradients (PHoG) first, then the Kullback-Leibler method is used to measure divergence. Only the highest-ranked matched classes are subjected to the classification procedure. Six distinct deep Convolution Neural Networks (DCNNs) were examined to achieve the final recognition. The networks examined in the study are AlexNet, VGG16, GoogleNet, Res50-Net, ResNeXt Net and DenseNet. The proposed multi-staging technique utilizing AlexNet $\mathrm{CNN}$ as a classifier has been observed to be effective in accurately identifying Arabic handwritten words.

Among other known contributions, a CNN-based approach is presented in [17] for recognition of handwriting in Arabic, English and French. The CNN model is employed to estimate the unigram, bigram and trigram frequency profiles for each input word. These estimated profiles are then matched with the true profiles using Canonical Correlation Analysis. 
The model reported an accuracy of $94.09 \%$ on Arabic handwriting samples. A Multi-dimensional, Long Short-Term Memory (MD-LSTM) architecture is presented for Arabic handwriting recognition in [18]. Sequence alignment is carried out using a connectionist temporal classification (CTC) layer. The system was evaluated on the collection of handwritten Arabic words in the IFN/ENIT dataset and reported an error rate of $8.57 \%$. [19] studied the impact of feature design on Arabic word recognition. Authors carried out a comparative study of a number of widely employed hand-crafted features with the features extracted automatically using MDLSTM. Experiments on the IFN/ENIT dataset led to the conclusion that recognition with an MDLSTM outperforms HMM based recognition with handengineered features.

After having discussed the well-known contributions to recognition of Arabic handwriting in both contemporary and historical documents, we now present the details of the proposed method in the next section.

Unlike the conventional approaches that rely only on either CNNs or RNNs, we propose in this paper to combine CNNs and RNNs with GRU for handwritten Arabic recognition in historical documents. Through recursive mechanisms, traditional RNNs process sequential data, however, for long-term sequential data, RNNs have severe problems. The GRU is designed to tackle these problems.

In addition to GRU, our proposed model uses CNN as an efficient feature extraction method. In this paper, we propose a deep CNN-GRU based solution to tackle the complexity of handwriting recognition in historical Arabic documents. To the best of our knowledge, this is the first time GRU networks are applied to historical Arabic documents and this is the first experiment that use a large number of Arabic subwords.

$$
\begin{aligned}
& z_{t}=\sigma\left(W_{z} \cdot\left[h_{t-1}, x_{t}\right]\right) \\
& r_{t}=\sigma\left(W_{r} \cdot\left[h_{t-1}, x_{t}\right]\right) \\
& h_{t}^{\tilde{t}}=\tanh \left(W \cdot\left[r_{t} * h_{t-1}, x_{t}\right]\right) \\
& h_{t}=\left(1-z_{t}\right) * h_{t-1}+z_{t} * h_{t}^{\tilde{t}}
\end{aligned}
$$

\section{Methods}

This section presents the details of the proposed subword recognition technique that relies on extraction of robust representations using convolutional layers followed by sequence modelling using Gated Recurrent Units (GRUs). An overview of the CNN-GRU architecture employed for recognition of subwords is illustrated in Figure 2. CNNs are known to be state-of-the-art feature extractors outperforming hand-engineered features on a number of classification tasks. Likewise, recurrent neural networks are known to effectively model sequential data.

While the basic RNNs are known to suffer from the vanishing gradient problem when modeling long-term dependencies, variants like LSTMs and GRUs are commonly employed. These variants rely on gates to regulate the flow of information from one time step to another. An LSTM cell typically has three gates, the forget gate, the input gate and the output gate. Compared to an LSTM, GRUs simplify the cell architecture and are known to be faster than LSTMs [20]. Through its update $\left(z_{t}\right)$ and reset $\left(r_{t}\right)$ gates in Equations 1,2, the GRU decides which information to keep and which to forget.

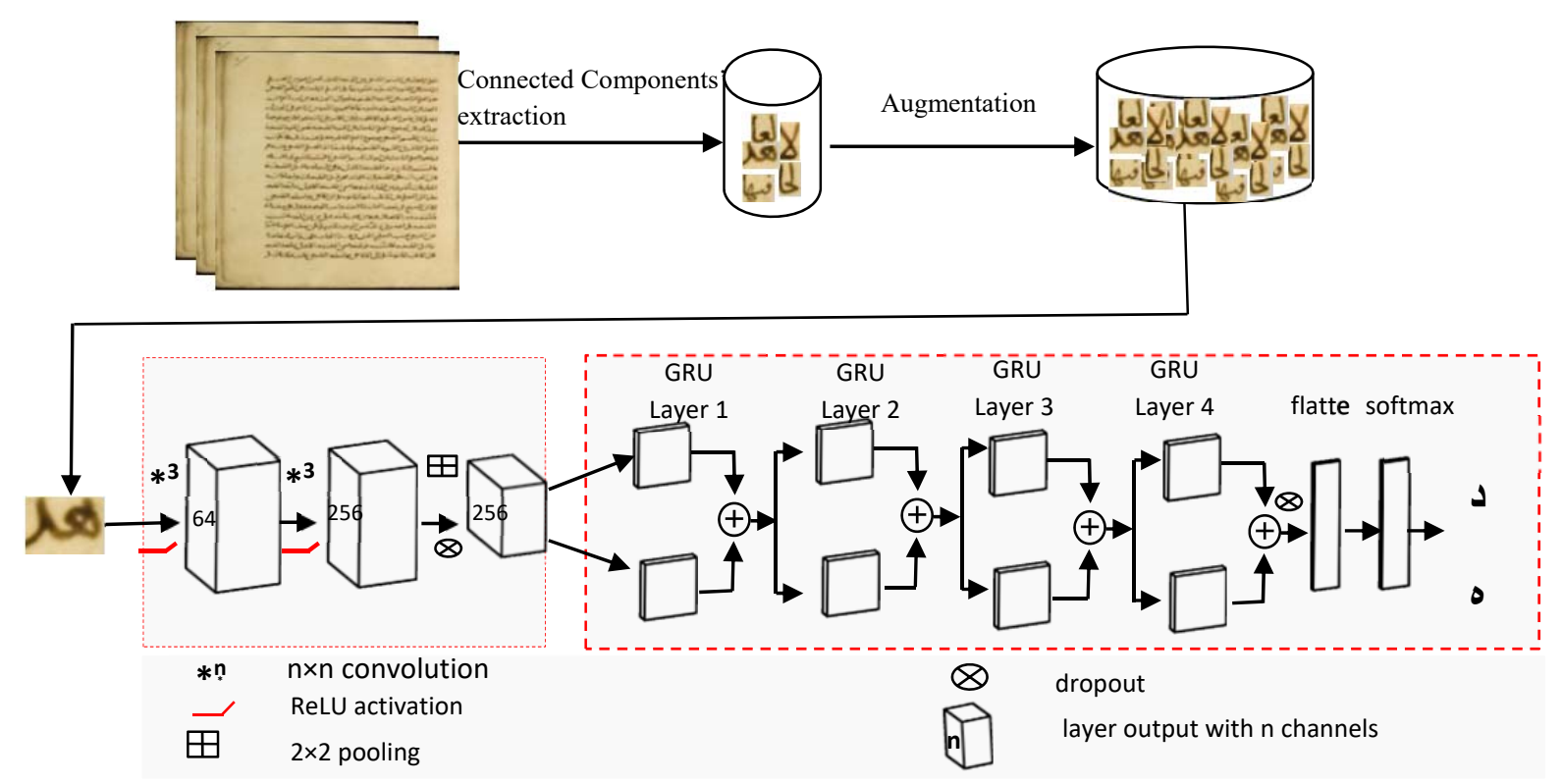

Figure 2. An overview of the CNN-GRU architecture for Arabic subword recognition. The Conv layers serve as features extractors while the stacked GRU layers serve as recognizer 
The employed architecture comprises of two convolutional layers. The input subword image $(40 \times 64 \times 3$ for images in the IBN-SINA dataset) is fed to the first convolutional layer with $64,3 \times 3$ filters producing an output of $40 \times 64 \times 64$. The feature maps of the first convolutional layer are fed as input to the second convolutional layer which produces an output of $40 \times 64 \times 256$. A max-pooling layer reduces the spatial dimensions of the output volume by 2 producing a volume of $20 \times 32 \times 256$. Batchnormalization is also added to the network to speed up training by normalizing the activations while a dropout layer is included to mitigate over-fitting. Prior to feeding the activations to the GRU layers, the data is reshaped to $20 \times 8192$. A stack of four Bidirectional GRU layers is added after the convolutional layers. Each layer scans the feature sequences in both the directions i.e., left to right as well as right to left. The outputs are then concatenated and fed to the next GRU layer. The output of the GRU layers is finally flattened and fed to a fully connected softmax layer for classification. The same architecture is employed for the VML-HD dataset, we only changed the input image size to $(110 \times 110)$.

The model is implemented in Tensorflow on TitanX GPU with 12 GB VRAM. Model was trained using ADAM optimizer, the learning rate was set to 0.001 while the batch size was set to 32 . The model was trained for 350 epochs.

\section{Experiments}

This section presents the details of the experiments along with the reported results. We first introduce the datasets employed in our study followed by a discussion on the results.

\subsection{Datasets}

One of the well-known datasets compiled for research on historical Arabic manuscripts is the IBNSINA dataset which contains 50 folios of ancient Arabic documents from KitabKashf alTamwihatfisharh al-Tanbihat (Figure 3.). Following the same experimental protocol as that of previous studies, we employ the first 40 folios for training and the last 10 folios for testing. Furthermore, we also ignore the dots similar to [13]. The second dataset, VML-HD, is recently published and is relatively much larger comprising of 680 pages from five different books written by different writers in diverse writing styles. Since there is no published work using the full version of this dataset yet, we employed $60 \%$ of images for training, $20 \%$ for validation and $20 \%$ in the test set.

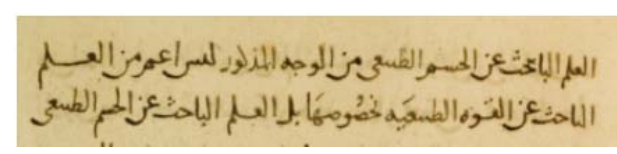

(a)

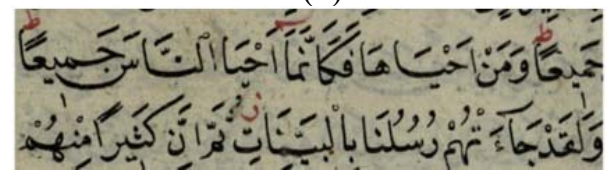

(b)

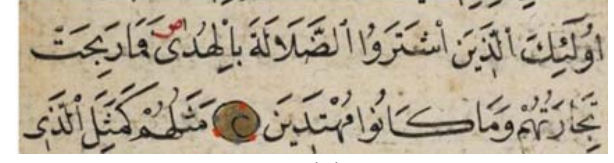

(c)

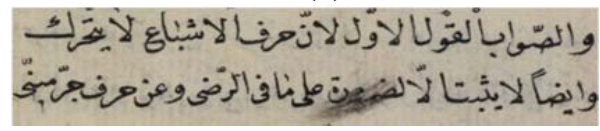

(d)

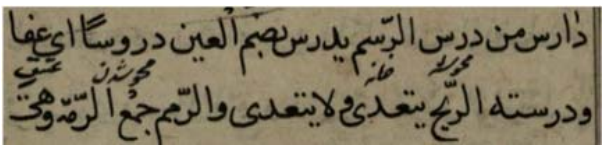

(e)

Figure 3. Samples of the datasets used. (a) Sample from IBN SINA dataset. (b) to (e) Samples from VML$H D$ dataset

The success of deep learning-based solutions is mostly linked with the amount of data employed for training. Most of the current research in object recognition using deep $\mathrm{CNNs}$ makes use of data augmentation to increase the size of training data and avoid over-fitting [21], [22]. Data augmentation can also be employed to address the class imbalance problem [23]. In our study, we employ shear transform and rotation (up to certain degrees) to augment the subword images. For each subword image in the IBN-SINA dataset, we generate 36 images resulting in a total of 179,027 images in 933 different categories. Since the VML-HD data is relatively larger and few classes have thousands of examples, we apply data augmentation only to classes having less than 10 samples. This results in a total of 374,161 subwords in 6187 different categories.

\subsection{Results \& Discussion}

The performance of the proposed CNN-GRU architecture on both the datasets is presented in Table 1. It can be observed from the reported recognition rates that though IBN SINA is a single writer dataset, the recognition rate is slightly lower $(96.13 \%)$ as compared to that on the VML dataset $(98.60 \%)$. 
Table 1. Recognition results in IBN SINA and VML-HD datasets

\begin{tabular}{|c|c|c|c|}
\hline Study & Method & Dataset & Accuracy \\
\hline $\begin{array}{l}\text { R. Alaasam et al. } \\
\text { [7] }\end{array}$ & $\mathrm{CNN}$ & $\begin{array}{l}\text { Part of } \\
\text { VML-HD }\end{array}$ & $81 \%$ \\
\hline $\begin{array}{l}\text { R. Alaasam et al. } \\
{[8]}\end{array}$ & $\mathrm{CNN}$ & $\begin{array}{l}\text { Part of } \\
\text { VML-HD }\end{array}$ & $97.82 \%$ \\
\hline $\begin{array}{l}\text { Chherawala et } \\
\text { al. [9] }\end{array}$ & $1-\mathrm{NN}$ & IBN SINA & $86.16 \%$ \\
\hline $\begin{array}{l}\text { Moghaddam et } \\
\text { al. [12] }\end{array}$ & SVMs & IBN SINA & $89.66 \%$ \\
\hline $\begin{array}{c}\text { Chherawala \& } \\
\text { Cheriet [10] }\end{array}$ & $1-\mathrm{NNs}$ & IBN SINA & $86.2 \%$ \\
\hline $\begin{array}{l}\text { Fouladi et al. } \\
\text { [13] }\end{array}$ & $\begin{array}{c}\text { Contour } \\
\text { Matching By } \\
\text { Alignment }\end{array}$ & IBN SINA & $91.08 \%$ \\
\hline $\begin{array}{l}\text { Proposed } \\
\text { Method }\end{array}$ & CNN and GRU & IBN SINA & $96.13 \%$ \\
\hline $\begin{array}{l}\text { Proposed } \\
\text { Method }\end{array}$ & $\mathrm{CNN}$ and GRU & $\begin{array}{l}\text { Full set of } \\
\text { VML-HD }\end{array}$ & $98.6 \%$ \\
\hline
\end{tabular}

This can be attributed to the relatively smaller training set in case of IBN SINA dataset as well as high degradation in the manuscripts. In some cases, small parts of subwords are lost leading to incorrect classification. When compared to other known methods evaluated on the IBN SINA dataset, a significant performance enhancement is observed as opposed to conventional classifiers like SVM and nearest neighbou

Likewise, the previous studies employing VML dataset used only a part of the dataset for evaluation of the proposed techniques. In our experiments, we employ the complete dataset with more than six thousand subword categories and the proposed model still outperforms the existing techniques. The high recognition rates reported on two different datasets support the potential application of such a system in automatic transcription of historical manuscripts. This could eventually lead to true digital libraries making them searchable for the end users.

Figure 4. illustrates the subwords categories which reported a recognition rate of less than $100 \%$. As discussed earlier, the degradation of manuscripts and incorrect segmentation of subwords are the major factors contributing to recognition errors as morphologically similar subword classes tend to overlap. As an example, the subword class ' $\mathrm{kr}$ ' in Table 2. has lost the upper stroke of the first letter making it resemble another subword ' $1 \mathrm{r}$ ' hence leading to an incorrect classification. Likewise, similar problems can occur with other subword classes resulting in incorrect recognition.
Table 2. Classification results. First three rows show incorrect classification while the last three rows show the correct classification

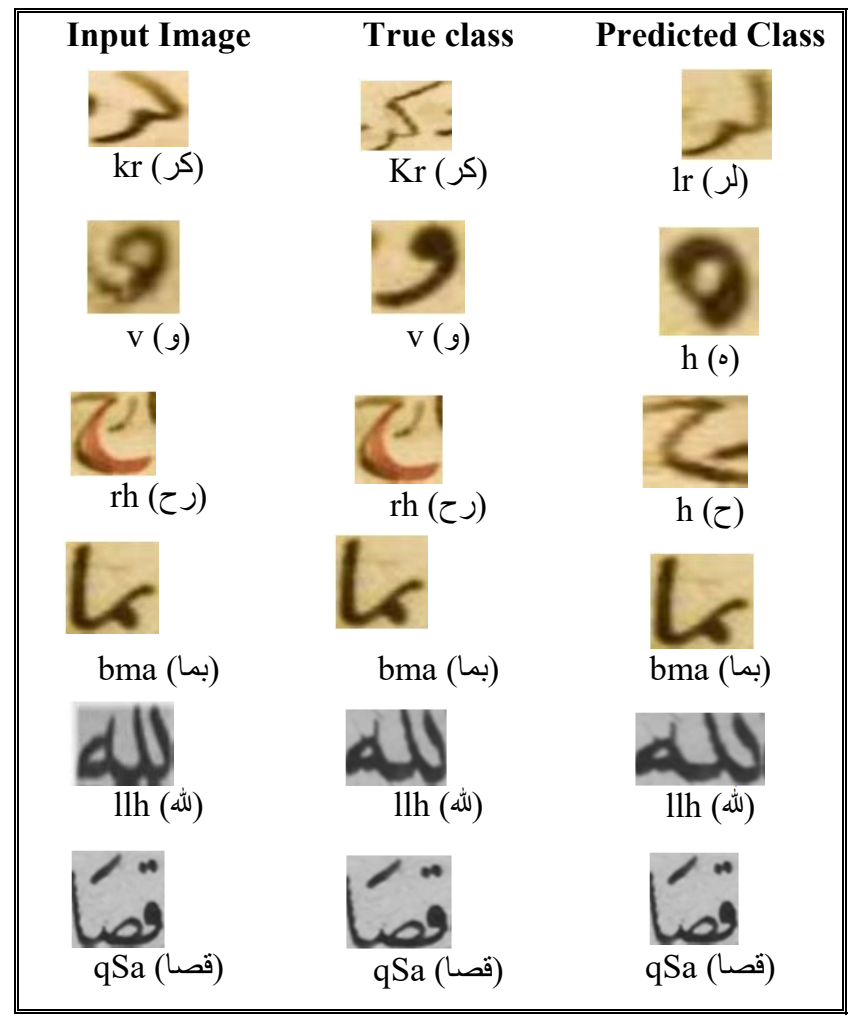

\section{Conclusion}

Recognition of Arabic handwriting from historical manuscripts is a challenging problem due to varying writing styles, document degradation and segmentation issues. The current study addressed this problem using a hybrid CNN-GRU model to recognize Arabic subwords extracted from historical documents. The proposed model was evaluated on two different datasets, IBN SINA and VML-HD and reported high recognition rates of $96.10 \%$ and $98.60 \%$ on the two datasets respectively. A comparison with existing techniques revealed the superiority of the proposed architecture in terms of recognition rates. The proposed solution can also be adapted to other cursive scripts like Persian, Kurdish and Urdu etc. In our subsequent endeavours on this subject, we aim to include a post-processing step to validate the predictions of the model. Furthermore, in addition to subwords, retrieval using complete words can also be added to allow more useful queries at the application layer. 


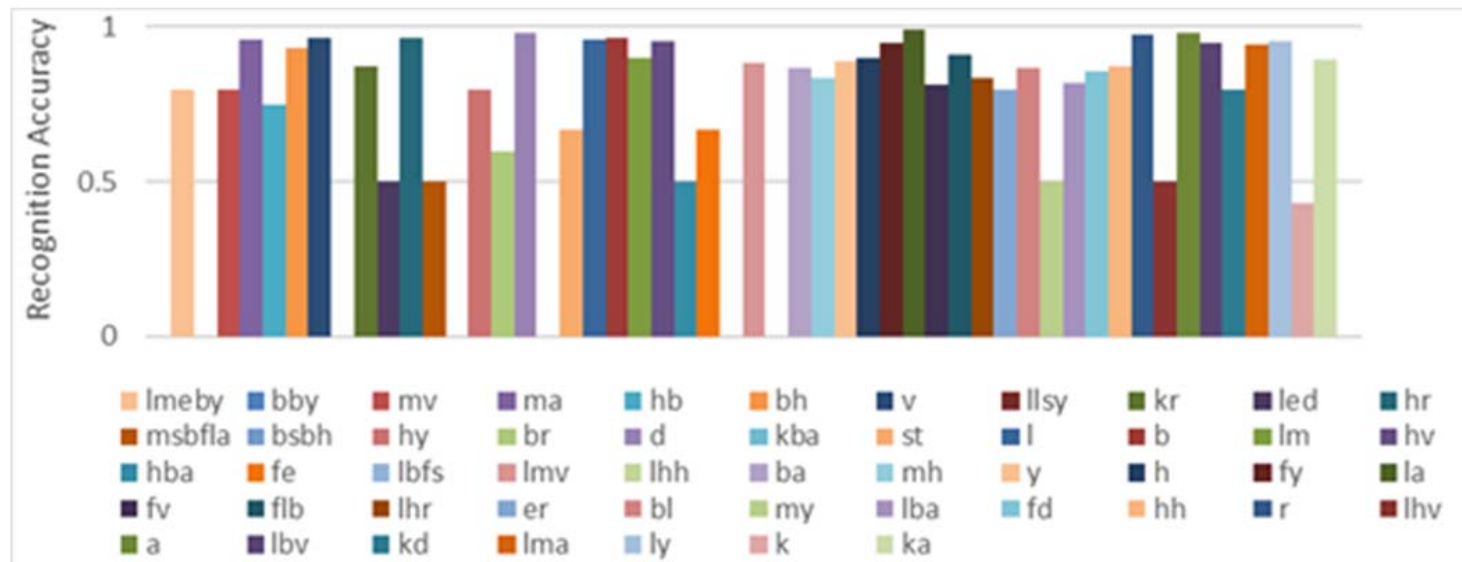

Figure 4. Categories of subwords that gained less than 100\% accuracy

\section{Acknowledgements}

This publication was made by NPRP grant \# NPRP $11 S$ - 0113 - 180276 from the Qatar National Research Fund (a member of the Qatar Foundation). The statements made herein are solely the responsibility of the authors.

\section{References}

[1]. Chherawala, Y., \& Cheriet, M. (2012). W-TSV: Weighted topological signature vector for lexicon reduction in handwritten Arabic documents. Pattern recognition, 45(9), 3277-3287.

[2]. Davoudi, H., Cheriet, M., \& Kabir, E. (2016). Lexicon reduction of handwritten Arabic subwords based on the prominent shape regions. International Journal on Document Analysis and Recognition (IJDAR), 19(2), 139-153.

[3]. Khedher, M. I., Jmila, H., \& El-Yacoubi, M. A. (2020). Automatic processing of Historical Arabic Documents: a comprehensive survey. Pattern Recognition, 100, 107144.

[4]. Pantke, W., Dennhardt, M., Fecker, D., Märgner, V., \& Fingscheidt, T. (2014, September). An historical handwritten arabic dataset for segmentation-free word spotting-hadara80p. In 2014 14th International Conference on Frontiers in Handwriting Recognition (pp. 15-20). IEEE.

[5]. Farrahi Moghaddam, R., Cheriet, M., Adankon, M. M., Filonenko, K., \& Wisnovsky, R. (2010, June). IBN SINA: a database for research on processing and understanding of Arabic manuscripts images. In Proceedings of the 9th IAPR International Workshop on Document Analysis Systems (pp. 11-18).

[6]. Kassis, M., Abdalhaleem, A., Droby, A., Alaasam, R., \& El-Sana, J. (2017, April). Vml-hd: The historical arabic documents dataset for recognition systems. In 2017 1st International Workshop on Arabic Script Analysis and Recognition (ASAR) (pp. 11-14). IEEE.

[7]. Alaasam, R., Kurar, B., Kassis, M., \& El-Sana, J. (2017, April). Experiment study on utilizing convolutional neural networks to recognize historical Arabic handwritten text. In 2017 1st International Workshop on Arabic script analysis and recognition (ASAR) (pp. 124-128). IEEE.
[8]. Alaasam, R., Barakat, B. K., \& El-Sana, J. (2018, March). Synthesizing versus augmentation for arabic word recognition with convolutional neural networks. In 2018 IEEE 2nd International Workshop on Arabic and Derived Script Analysis and Recognition (ASAR) (pp. 114-118). IEEE.

[9]. Chherawala, Y., Wisnovsky, R., \& Cheriet, M. (2011, September). TSV-LR: topological signature vectorbased lexicon reduction for fast recognition of premodern Arabic subwords. In Proceedings of the 2011 Workshop on Historical Document Imaging and Processing (pp. 6-13).

[10]. Chherawala, Y., \& Cheriet, M. (2014). Arabic word descriptor for handwritten word indexing and lexicon reduction. Pattern recognition, 47(10), 3477-3486.

[11]. Pechwitz, M., Maddouri, S. S., Märgner, V., Ellouze, N., \& Amiri, H. (2002, October). IFN/ENIT-database of handwritten Arabic words. In Proc. of CIFED (Vol. 2, pp. 127-136). Citeseer.

[12]. Moghaddam, R. F., Cheriet, M., Milo, T., \& Wisnovsky, R. (2012, July). A prototype system for handwritten sub-word recognition: Toward Arabicmanuscript transliteration. In 2012 11th International Conference on Information Science, Signal Processing and their Applications (ISSPA) (pp. 11981204). IEEE.

[13]. Fouladi, K., Araabi, B. N., \& Kabir, E. (2014). A fast and accurate contour-based method for writerdependent offline handwritten Farsi/Arabic subwords recognition. International Journal on Document Analysis and Recognition (IJDAR), 17(2), 181-203.

[14]. Al-Jawfi, R. (2009). Handwriting Arabic character recognition LeNet using neural network. Int. Arab J. Inf. Technol., 6(3), 304-309.

[15]. Amrouch, M., \& Rabi, M. (2017, April). Deep neural networks features for Arabic handwriting recognition. In International Conference on Advanced Information Technology, Services and Systems (pp. 138-149). Springer, Cham.

[16]. Ghanim, T. M., Khalil, M. I., \& Abbas, H. M. (2020). Comparative study on deep convolution neural networks DCNN-based offline Arabic handwriting recognition. IEEE Access, 8, 9546595482. 
[17]. Poznanski, A., \& Wolf, L. (2016). Cnn-n-gram for handwriting word recognition. In Proceedings of the IEEE conference on computer vision and pattern recognition (pp. 2305-2314).

[18]. Graves, A. (2012). Offline arabic handwriting recognition with multidimensional recurrent neural networks. In Guide to OCR for Arabic scripts (pp. 297-313). Springer, London.

[19]. Chherawala, Y., Roy, P. P., \& Cheriet, M. (2013, August). Feature design for offline Arabic handwriting recognition: handcrafted vs automated?. In 2013 12th International Conference on Document Analysis and Recognition (pp. 290-294). IEEE.

[20]. Zhang, X. Y., Yin, F., Zhang, Y. M., Liu, C. L., \& Bengio, Y. (2017). Drawing and recognizing chinese characters with recurrent neural network. IEEE transactions on pattern analysis and machine intelligence, 40(4), 849-862.
[21]. Cireşan, D. C., Meier, U., Gambardella, L. M., \& Schmidhuber, J. (2010). Deep, big, simple neural nets for handwritten digit recognition. Neural computation, 22(12), 3207-3220.

[22]. Krizhevsky, A., Sutskever, I., \& Hinton, G. E. (2012). Imagenet classification with deep convolutional neural networks. Advances in neural information processing systems, 25, 1097-1105.

[23]. Afzal, S., Maqsood, M., Nazir, F., Khan, U., Aadil, F., Awan, K. M., ... \& Song, O. Y. (2019). A data augmentation-based framework to handle class imbalance problem for Alzheimer's stage detection. IEEE Access, 7, 115528-115539. 